\title{
HOST STARVATION DECREASES PARASITE LOAD AND MEAN HOST SIZE IN EXPERIMENTAL POPULATIONS
}

\author{
Katja Pulkkinen ${ }^{1}$ And Dieter Ebert ${ }^{2}$ \\ Zoological Institute, University of Basel, Rheinsprung 9, 4051 Basel, Switzerland
}

\begin{abstract}
While host stress in vertebrate populations has often been linked to outbreaks of epidemics, which are attributed to the immuno-compromise of the stressed hosts, no predictions have been made about the response of invertebrate host populations to stressful conditions. Experiments conducted on individual invertebrate hosts, however, suggest that starved hosts may be a poor resource for parasites and that heavily infected old hosts may be more susceptible to stress, causing parasite populations to decline when their host population faces food shortages. In this epidemiological experiment, we exposed infected and uninfected Daphnia magna populations, which had been kept for many generations under a constant high food supply, to reduced food resources. Using the microsporidian gut parasite Glugoides intestinalis, which is exclusively horizontally transmitted, we tracked changes in parasite and host population size as well as host body length to elucidate how food shortages for the hosts influence host and parasite population dynamics. In both infected and uninfected populations, food shortage led to an approximately equal reduction in host density and changes in host body length distribution. Large hosts suffered a higher mortality than smaller hosts, which significantly reduced the mean body length in the host populations; however, this change was stronger in the infected populations and went handin-hand with a reduction in parasite spore load (a measure of intensity of infection) and prevalence. This effect disappeared after six weeks of food shortage, when the populations reached a new equilibrium. Our results indicate that in this system food stress impairs parasite spread and that host mortality is an important factor in regulating parasite abundance at the population level.
\end{abstract}

Key words: Daphnia magna; experimental epidemiology; Glugoides intestinalis; microsporidia; stress-induced immunosuppression; stress-induced mortality.

\section{INTRODUCTION}

Conventional wisdom holds that when living conditions deteriorate for hosts, for example during periods of overcrowding or food shortage, infectious diseases spread more rapidly, leading to an increase in parasite population size (Anderson and May 1981, Lloyd 1995). Laboratory experiments have established that malnutrition can lead to higher parasite loads in individual hosts (Wakelin 1989, Lloyd 1995). At the population level, however, this contention is based mostly on observations of vertebrate animals, where overcrowding and malnutrition are often coupled with high burdens of parasites and high mortality of infected animals (Anderson and May 1981, Gulland 1992, 1995). A plausible explanation for this observation is that stress impairs the defense mechanisms of the hosts and leads to an increase in parasite within-host growth and transmission. However, the size of a parasite population is

Manuscript received 18 March 2003; accepted 26 June 2003; final version received 28 July 2003. Corresponding Editor: J. T. Wootton.

${ }^{1}$ Present address: Department of Biological and Environmental Sciences, University of Jyväskylä, P.O. Box 35, 40014 University of Jyväskylä, Finland.

${ }^{2}$ Present address: Ecology and Evolution, Department of Biology, Fribourg University, Chemin du Musee 10, CH-1700 Fribourg, Switzerland. determined by a balance between the increase of parasites via transmission and within-host growth and the removal of parasites via parasite and host mortality (Anderson 1978, 1979a, Anderson and May 1978). If host stress has a stronger effect on parasite removal than on their growth and transmission, then the net effect of stress may be a decline in prevalence rather than an increase. This can occur when stress-induced mortality increases among infected hosts (Anderson $1979 a$ ). The balance of factors leading to an increase or a decrease of the parasite population size is likely to depend on the host-parasite system (Anderson and May 1979).

Research on vertebrates has shown that malnutrition can suppress immunocompetence, resulting in higher parasite loads due to increased within-host growth (see, e.g., reviews by Wakelin [1989] and Lloyd [1995]) and leading, in turn, to increased transmission rates. Invertebrates differ from vertebrates with regard to their immune system (e.g., they lack acquired immunity; Briggs et al. 1995) and their generation times and life spans are much shorter than those of vertebrates, and infections are usually chronic (i.e., there is no recovery from infections). Further, as a considerable proportion of host biomass is often converted into parasite biomass (e.g., parasitoids, baculoviruses), many parasites of invertebrates depend strongly on host biomass and re- 
sources. Therefore, host stress may have a stronger negative effect on parasite within-host growth and survival (via host deaths) and a more important role in determining the size of the parasite population in invertebrates. In the present study, we tested experimentally how resource limitation imposed on invertebrate host populations affects both host and parasite populations. Our prediction was that food stress would induce high mortality in heavily infected host individuals, thus removing a large part of the parasite population and leading to a net decrease in the parasite load in the host population.

As a model system we used the freshwater crustacean Daphnia magna and a horizontally transmitted microsporidian parasite that reproduces directly within the host. The Daphnia-microparasite system is especially suitable for studying epidemiological questions because it enables one to simultaneously manipulate a large number of replicate populations and to compare the effects of perturbation to the equilibrium conditions. In the absence of predators or parasites, the Daphnia's mean population size is determined mainly by the rate of feeding (Slobodkin 1954). At constant food supply, the population settles to an equilibrium in which the total number of animals, reproductive rates, growth rates, and size-frequency distribution remain largely constant over time (Slobodkin 1954, Perrin et al. 1992). Epidemiological models predict that when a parasite persists in a host population under a certain parameter space, it regulates the host population at or around an equilibrium level (Anderson 1978, 1979b). Since Daphnia can be maintained in laboratory conditions such that they reproduce only asexually, it is possible to exclude effects of host genetics on disease dynamics and to concentrate purely on epidemiological questions. This corresponds to the Daphnia's natural situation, as they reproduce mostly clonally during summer; in small water bodies, especially, the whole population may consist of only one or a few clones (Ebert et al. 2002). In many zooplankton communities, scarcity of food is considered to be a major controlling force (Lampert and Muck 1985), and natural populations of Daphnia frequently suffer from population crashes after food shortages (Tessier and Goulden 1982, Ghilarov 1985). Microsporidian parasites are the most common parasites of Daphnia in natural populations (Stirnadel and Ebert 1997, Ebert et al. 2001). Therefore the question of how nutrition influences parasite epidemiology is also relevant for real Daphnia populations.

In Daphnia, resistance to starvation has been shown to depend on the energy reserves and thus on size of the individual (Tessier and Goulden 1982, 1987, Tessier et al. 1983, Tessier and Consolatti 1989, Perrin et al. 1992). We therefore predicted that a food shortage in uninfected populations would increase the mortality of young, small Daphnia. However, the effect of parasites on host survival correlates positively with the intensity of infection (Anderson 1979a, Anderson and May 1979, Ebert et al. 2000b). In infected Daphnia populations the largest animals have the highest parasite load due to their higher filtering rate and accumulation of parasites through time (Ebert 1994, Mangin et al. 1995, Stirnadel and Ebert 1997). Therefore, our second prediction was that, as opposed to uninfected populations, the large animals in infected populations would be among the first to die in a food shortage. The high mortality of large animals would lead to a decrease in the mean body length of the Daphnia and to a removal of a large part of the parasite population. Furthermore, it has been shown that the level of host nutrition affects parasite growth in Daphnia, so that parasites grow more slowly in hosts kept on low diet (Ebert et al. 2000b, Bittner et al. 2002). This would further inhibit parasite population from increasing in a starved host population. However, we predicted that when food deprivation continued for some time, the populations would reach a new equilibrium at the lower food level, with the population numbers remaining low, but the body length distribution returning to approximately prestarvation levels. In infected populations, this could either lead to an increase in the mean parasite load or, because of the decrease in the density of the host population, it could act in an opposite direction (Anderson and May 1979). Thus, the final outcome was not easily predictable. In this paper we present the results of an epidemiological experiment in which well-fed uninfected or infected Daphnia populations were subjected to a food shortage. The aim of the study was to understand the relative role of the forces that influence host and parasite population size and host body length structure and to use these population-level findings to expand our knowledge of host-parasite interactions as observed on the individual level.

\section{Methods}

\section{The host-parasite system}

The model system we used in the experiment consisted of a freshwater crustacean, Daphnia magna Straus (Crustacea: Cladocera), and its obligatory parasitic microsporidian, Glugoides intestinalis (Microspora: Glugeidae; Larsson et al. 1996). D. magna is a cyclic parthenogen that can be maintained in the laboratory in clonally reproducing populations. Males and fertilized resting eggs are produced when conditions deteriorate (e.g., due to crowding). Under laboratory conditions, resting eggs usually do not hatch without dormancy, and thus the genetic composition of a monoclonal Daphnia population does not change during experimentation.

Due to the small size of $D$. magna (maximum length $4 \mathrm{~mm}$ ), large populations can be maintained in small vessels in the laboratory, enabling us to maintain many populations under controlled conditions. The vessels are small enough to exclude spatial heterogeneity in 
the populations; still the population numbers are large enough for disease dynamics to occur. With a constant food supply and without parasites, Daphnia populations reach an equilibrium population size, representing a carrying capacity determined by the food quantity (Slobodkin 1954, Perrin et al. 1992, Ebert et al. 2000a). At $20^{\circ} \mathrm{C}$, the generation time of D. magna is between 8 and 16 days (depending on resources available).

G. intestinalis (Larsson et al. 1996) is an intracellular parasite in the gut epithelium of Daphnia that infects its host through waterborne spores. It reproduces directly, and transmission is horizontal between hosts (Ebert 1995). It best fits the definition of a microparasite used in epidemiological models (May and Anderson 1979). Hosts do not recover from infection (Ebert et al. 2000a). The parasite is transmitted from live hosts and dead hosts (Ebert 1995), but transmission through dead hosts is less likely (D. Ebert, personal observation). Reinfection of the same host with spores released with the feces due to ingestion during filter feeding is common (Ebert 1995). The number of parasite spores found inside the host is directly correlated with the parasite's transmission success (Ebert 1994). Exposure to larger doses (higher concentration of the waterborne spores) leads to higher parasite loads (Ebert and Mangin 1997).

The Daphnia clone used in the experiment originated from a pond in North Germany near Gaarzerfeld. It was collected in 1997 and maintained in the laboratory since. The G. intestinalis strain used in the experiment was isolated from this host clone and propagated in the same host genotype in the laboratory.

\section{Population dynamics experiment}

We cultured Daphnia populations in glass jars filled with $1 \mathrm{~L}$ of artificial freshwater medium (Klüttgen et al. 1994) that we modified by using only one-twentieth of $\mathrm{SeO}_{2}$ concentration and adding one-fifth water from a local well. We fed the animals daily with suspension of the green algae Scenedesmus gracilis grown in chemostats. We kept the cultures in an air-conditioned room at $20^{\circ} \mathrm{C}$ with a $16: 8$ light:dark period and randomized the position of the jars every week.

We bred uninfected Daphnia in the laboratory and began the experiment by distributing them evenly into 40 populations of 250 animals each so that the body length distribution was as similar as possible among all populations. We randomly chose 20 populations to be infected with $G$. intestinalis. To each of these populations we added 20 infected adult females within a net enclosure for $1 \mathrm{wk}$. The mesh size of the net $(250$ $\mu \mathrm{m})$ kept the infected and uninfected Daphnia from mixing, but allowed algae and parasite spores to pass through. The nets were shaken every day to maximize the transport of spores into the culture. To the 20 uninfected populations, we added uninfected Daphnia in the net enclosures.
We removed one-fourth of the medium together with the Daphnia from each population every week after mixing it in a Folsom plankton sample divider, and we replaced the removed water with fresh medium. We counted the animals removed every week and measured them for body length (from the top of the head to the base of the tail spine) every other week. All animals used to determine parasite spore load and for the experiment for time until death under complete starvation were from this removed portion. We kept all populations well fed $\left(110 \times 10^{6}\right.$ algae cells $\left.\cdot \mathrm{jar}^{-1} \cdot \mathrm{d}^{-1}\right)$. When approximate population size equilibrium was reached, we took a subsample of the animals from the weekly removed portion to determine spore load in the populations. These samples, and all later spore samples, were frozen for later counting. From this point onwards, we randomly assigned 10 of the 20 uninfected and 10 of the 20 infected populations to the food reduction treatment. Starved populations received oneeighth of the standard food ration $\left(13.8 \times 10^{6}\right.$ algae cells $\left.\cdot \mathrm{jar}^{-1} \cdot \mathrm{d}^{-1}\right)$. The other populations were fed as before. One week after the food reduction treatment began, we took a second sample to ascertain spore load. We continued the food reduction treatment until those populations settled to equilibrium at the lower food level. We then sampled the infected populations to determine the spore load for the third time.

Once per month we checked populations for the presence (infected populations) or absence (uninfected populations) of infection by dissecting five large females from each population and checking for spore clusters in the guts. All infected populations remained infected until the end of the experiment, although one was lost accidentally. We also discovered one of the uninfected populations to be infected on week 21 and therefore excluded it from all analyses.

\section{Spore load dynamics}

To determine mean parasite load, we took 10 animals per sampling from each population. The animals were taken in proportion to the size distribution of the animals in each population. The animals were measured for body length and placed singly in $50 \mu \mathrm{L}$ of distilled water in micro test tubes and frozen for later spore counting. Spore counting was done by grinding up the whole Daphnia in the $50 \mu \mathrm{L}$ of the water it was frozen in and counting the spores in a bacterial counting chamber (Thoma ruling). Because this method has a detection threshold of $\sim 200$ spores per animal, it is possible that some early infections in animals with only a few spore clusters were missed, perhaps leading to a slight underestimation of prevalence. However, since the heavily infected animals had more than 100000 spores, spore load counts were not affected. As large animals contribute more volume to the total volume in which we counted the spores, we corrected the sample volume for this body length effect. We calculated the volume of each animal by converting their body length ac- 
cording to a power equation that we obtained by weighing 96 Daphnia magna with a size range from 0.9 to $3.7 \mathrm{~mm}$ and assuming that the density of the Daphnia equals that of water. The power equation used was volume $=0.2418 \times$ length $^{2.593}$.

\section{Mortality during food reduction in the population experiment}

In addition to population censuses, we tracked mortality in the populations directly by collecting the dead animals from all the populations daily during days 3 to 7 after the food reduction treatment was begun. We measured the length of the dead animals to see if the size distribution of dying animals differed according to treatments. Since the size frequency distribution was bimodal, we divided the animals into two size classes, 0.9-2.0 $\mathrm{mm}$ (small) and 2.1-3.7 $\mathrm{mm}$ (large), which corresponds approximately to nonreproducing and reproducing Daphnia size classes. In each population, we calculated the probability of death within both size classes for each day and calculated means separately for all treatments. We calculated the number alive each day by subtracting the cumulative number of dead animals from the number alive before food reduction. Since we calculated the probability of death starting from those alive on day zero, the values we obtained for each day are a slight underestimation of the real values, but the proportions of the probabilities between days are not affected.

\section{Experiment for time until death under complete starvation}

To test for size-specific mortality in our populations, we measured the time until death under complete starvation for different sizes of individual animals. We took five animals from each of the 40 populations on week 14 and placed them singly in $100-\mathrm{mL}$ jars in artificial medium. The medium was filtered through a $0.2-\mu \mathrm{m}$ pore size filter to remove any bacterial growth that might serve as food for the Daphnia. The medium was replaced every day. At the beginning of this starvation experiment, we measured the body length of the animals. We recorded the survival of the animals daily and ran the experiment until the death of the last experimental animal. We could not verify the infection status of the animals taken from infected populations, since in most cases they degraded too quickly after their death. However, from other parasite checks in our experimental populations we know that in an infected population most hosts carry the parasite. We calculated the mean time to death for both uninfected and infected animals in the two size classes (small and large).

\section{Data analysis}

The effect of food reduction treatment and infection on population counts and mean body lengths of Daphnia were analyzed with GLM repeated-measures ANOVA using log-transformed data. Week was included as a within-subject factor and infection status and food reduction treatment as between-subject factors. In this analysis, a statistically significant food reduction effect in itself is not an indication of the effect of food reduction treatment; instead this is indicated only by a statistically significant interaction between food reduction and week after the food reduction treatment began in week 21 . In these analyses, we included only measurements from week 15 onwards, because the marked fluctuations in population counts before this time were likely to be caused by the starting conditions. Since the circularity assumptions of the variance-covariance matrix (von Ende 1993) were not met, we used Huynh-Feldt corrected significance levels (Potvin and Lechowicz 1990). Among the four treatments, the skewness and kurtosis values of the Daphnia populations after food reduction treatment were compared with ANOVA on weeks 22 and 27 separately for each week. We used two parameters to measure the spore load in infected populations: mean spore load and prevalence. Of these two, prevalence is most closely related to transmission success in microparasites. We calculated the mean spore load of a population as the mean of the spore load of the 10 animals subsampled, including uninfected animals and prevalence as a proportion of infected animals in the subsample. The effect of the food reduction treatment on mean parasite load and prevalence of infection were tested with $t$ tests between well-fed and starved populations separately for each sampling point. The probabilities of infection at a certain body length for individual Daphnia were compared between the starved and well-fed groups separately for each of the three sampling points with a logistic regression. The data on the probabilities of death during the 3-7 d after food reduction were not normally distributed, so we analyzed them using nonparametric Kruskal-Wallis tests. We compared the probability of death between well-fed and starved populations in the two size classes and between the size classes among well-fed and starved populations. We also compared uninfected and infected animals within each of the four groups. The mean time to death of the individual animals under complete starvation was tested between small and large animals separately for uninfected and infected animals with Mann-Whitney $U$ tests, because the data were not normally distributed. We used Bonferroni correction to adjust the levels of statistical significance for multiple tests. All statistical analyses were done with SPSS 10.0 (SPSS 1999). In the figures included in this paper, the well-fed animals are always depicted with open symbols and the starved animals with filled symbols.

\section{Results}

\section{Population dynamics experiment}

Population densities fluctuated around 200 individuals (Fig. 1A). At week 21, we considered the popu- 

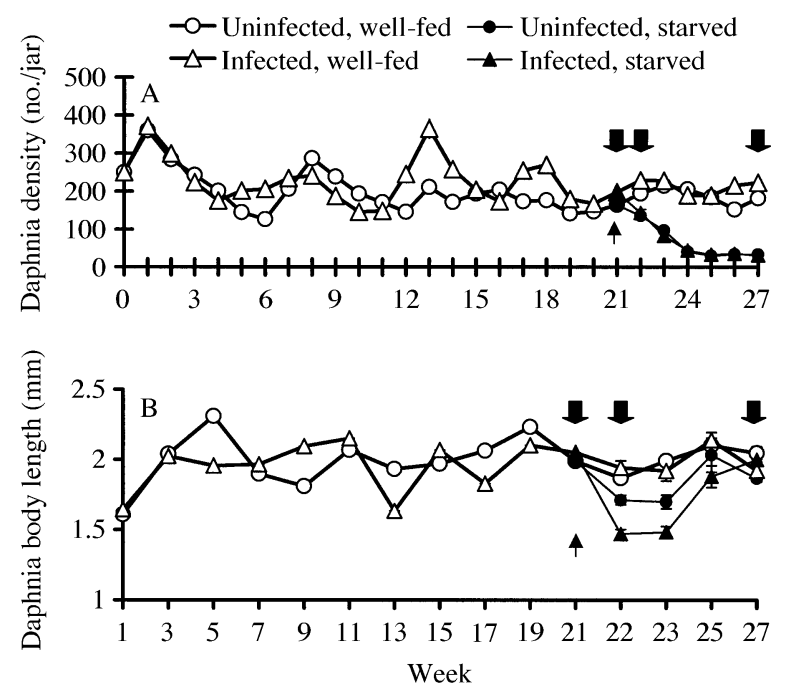

FIG. 1. (A) Population dynamics and (B) body lengths of Daphnia magna in uninfected populations and populations infected with Glugoides intestinalis (means $\pm 1 \mathrm{SE}$ ) before (until week 21) and after food reduction treatment. The start of the food reduction treatment is indicated with an arrow from below, and the sampling for spore counts is indicated with block arrows. Note that the scales for week ( $x$-axis) differ slightly.

lations to have reached equilibrium and took the first samples for spore load from the infected populations. Immediately after this, we assigned half of both uninfected and infected populations to the food reduction treatment (one-eighth of the normal food ration) and took a second sample 1 wk later (week 22). In the populations that were continuously well fed, the densities kept fluctuating around 200 animals per jar, while the starved populations settled to around 20 animals. We took a third sample for determining spore load at week 27 (Fig. 1A). The interaction between food reduction and week was highly significant (repeatedmeasures ANOVA, within-subjects factors, week $\times$ food reduction, $F=112.9$, df $=9.6,327.8, P<0.001$ ), and contrasting between the levels of within-subjects factor (week) revealed that this interaction became significant only after the onset of the food reduction treatment at week 21. The effect of parasitism on the population size was also significant (between-subjects effects, $F=20.0$, df $=1,34, P<0.001$ ), but the infected populations contained slightly more animals.

During the two weeks after the food reduction treatment was begun (weeks 22 and 23), mean body length of Daphnia decreased in the starved populations as compared to the well-fed populations (Fig. 1B, Table 1 , within-subjects contrasts). However, when the food reduction had continued for $4 \mathrm{wk}$ (approximately two host generations; week 25), the mean body length returned to its prestarvation level (Fig. 1B, Table 1, within-subjects contrasts). The mean body length of Daphnia was also shorter in infected than in uninfected populations (Table 1, between-subjects effects). After food reduction was begun, during week 22 , the decrease in mean body length was greater for infected than for uninfected populations (Fig. 1B) as indicated by the contrasting of the three-way interaction between week, food reduction, and infection (Table 1, within-subject contrasts).

TABle 1. Repeated-measures ANOVA for the mean length of Daphnia magna.

\begin{tabular}{lrrrr}
\hline \multicolumn{1}{c}{ Source } & $\mathrm{df}$ & $\mathrm{MS}$ & \multicolumn{1}{c}{$F$} & \multicolumn{1}{c}{$P$} \\
\hline Between subjects & & & & \\
Infection (I) & 1 & 0.035 & 18.662 & $<0.001$ \\
Food reduction (R) & 1 & 0.124 & 65.624 & $<0.001$ \\
I $\times$ R & 34 & 0.002 & 0.927 & 0.342 \\
Error & & 0.002 & & \\
Within subjects $\dagger$ & & & \\
Week (W) & 4.780 & 1.077 & 27.173 & $<0.001$ \\
W $\times$ I & 4.780 & 0.167 & 4.203 & 0.002 \\
W $\times$ R & 4.780 & 0.320 & 8.078 & $<0.001$ \\
W $\times$ I $\times$ R & 4.780 & 0.117 & 2.943 & 0.016 \\
Error & 162.522 & 0.040 & & \\
& & & & \\
& & Source & & W \\
Within-subjects contrasts & $\mathrm{W}$ & $\mathrm{W} \times \mathrm{I}$ & $\mathrm{W} \times \mathrm{R}$ & $\mathrm{R}$ \\
\hline Week 17 vs. week 15 & 0.064 & $<0.001$ & 0.293 & 0.627 \\
Week 19 vs. previous & $<0.001$ & 0.189 & 0.415 & 0.732 \\
Week 21 vs. previous & 0.481 & 0.050 & 0.665 & 0.529 \\
Week 22 vs. previous & $<0.001$ & 0.432 & $<0.001$ & $<0.001$ \\
Week 23 vs. previous & $<0.001$ & 0.095 & $<0.001$ & 0.239 \\
Week 25 vs. previous & 0.039 & 0.864 & 0.581 & 0.406 \\
Week 27 vs. previous & 0.967 & 0.139 & 0.161 & 0.002 \\
\hline
\end{tabular}

Notes: The $P$ values for the within-subjects contrasts are given for the factors having statistically significant within-subjects effects. The contrast is "difference," which compares each level to the mean of all previous levels.

$\dagger$ Huynh-Feldt corrected. 


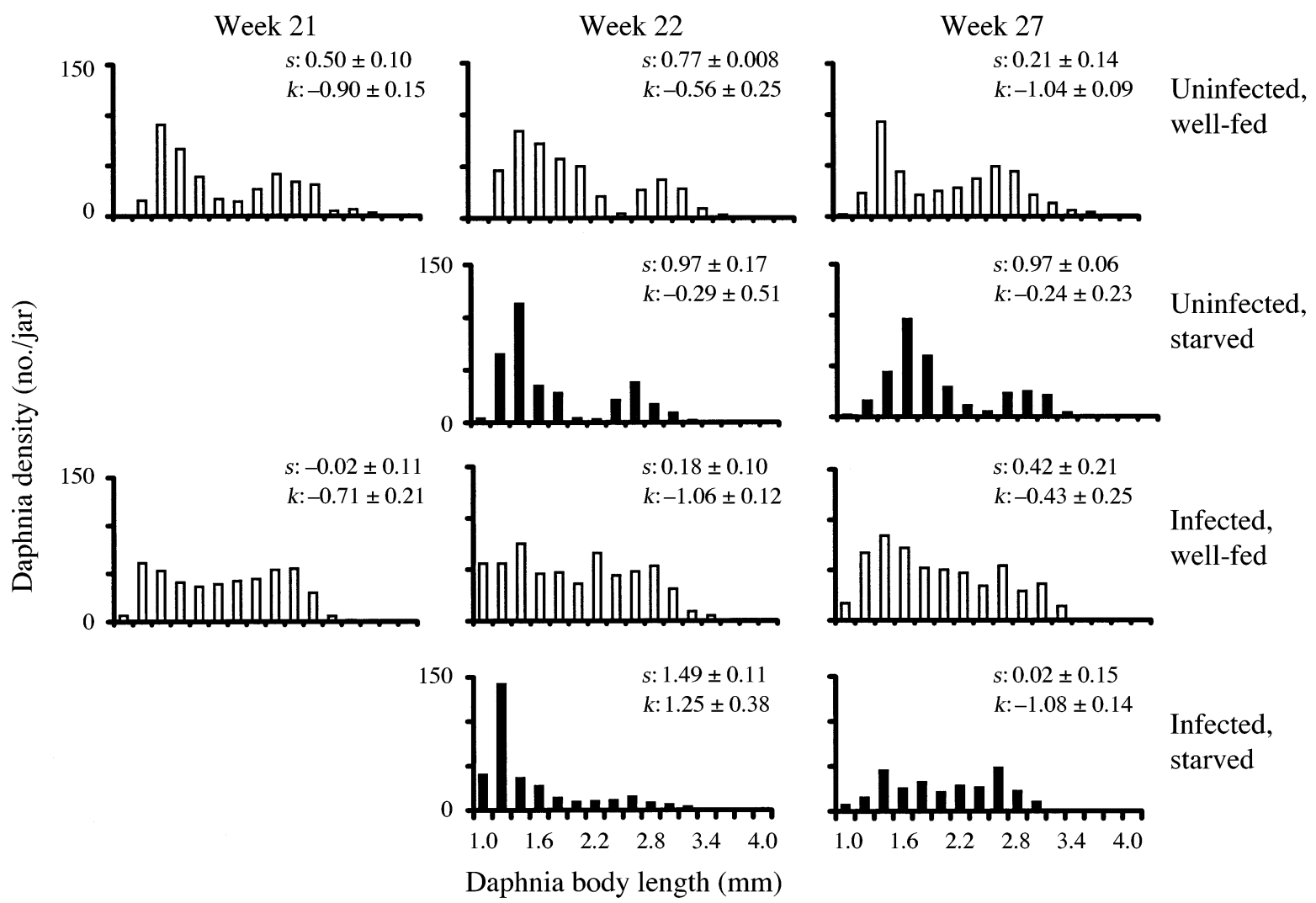

FIG. 2. Length distributions of uninfected Daphnia magna populations and populations infected with Glugoides intestinalis (sums of replicates) before food reduction treatment (week 21) and 1 and 6 wk (weeks 22 and 27, respectively) after the beginning of food reduction treatment. The numbers within figures show skewness $(s)$ and kurtosis $(k)$ values of the Daphnia populations at each time point (means $\pm 1 \mathrm{SE}$ ). Positive skewness values indicate that populations are skewed to the right, i.e., most of the animals are smaller than the mean of the population. Negative kurtosis values indicate that the distribution is flatter than the normal distribution, and positive values show that the distribution is more peaked than the normal distribution.

In addition to a decrease in mean body length, the shape of the body length distribution of Daphnias changed under food reduction (Fig. 2). After $1 \mathrm{wk}$, the distributions in the starved populations became more positively skewed as compared to the well-fed populations, i.e., there were more small animals than large animals in the populations (Fig. 2; ANOVA, $F=36.7$, $\mathrm{df}=1,34, P<0.005$, level of significance Bonferroni adjusted for two tests, $\alpha=0.001 / 2)$. The increase in skewness was significantly higher for the infected starved populations than for the uninfected starved populations (ANOVA, food reduction treatment $\times$ infection status interaction, $F=19.6$, df $=1,34, P<$ $0.005)$. Kurtosis also increased in the starved populations after $1 \mathrm{wk}$ as compared to the well-fed populations (Fig. 2; ANOVA, $F=13.5$, df $=1,34, P<0.005$ ). The change was much more marked in the infected starved populations (ANOVA, food reduction treatment $\times$ infection status interaction, $F=8.5, \mathrm{df}=1$, $34, P<0.025)$. After $6 \mathrm{wk}$, the starved populations returned to the body length distribution before treatment (Fig. 2), showing in week 27 no difference in skewness or kurtosis from the well-fed populations
(ANOVA, $F=1.4$, df $=1,34, P=0.251$ for skewness and $F=0.1$, df $=1,34, P=0.721$ for kurtosis).

\section{Spore load dynamics}

Before food reduction (week 21) neither the mean spore load nor the prevalence (proportion of infected hosts) differed significantly in the infected populations later allocated to the food reduction treatment or not (Fig. 3; $t$ test, $t=1.6$, df $=17, P=0.139$ for mean spore load and $t=1.9$, df $=17, P=0.072$ for mean prevalence). After 1 wk of food reduction (week 22), both the mean spore load and the prevalence decreased significantly in the starved populations compared to the well-fed populations (Fig. $3 ; t$ test, $t=3.9$, df $=$ $11.2, P<0.003$ for mean spore load and $t=3.9$, df $=17, P<0.003$ for prevalence; levels of significance Bonferroni adjusted for three tests, $\alpha=0.01 / 3$ ). In week 27, there was neither a significant difference in the mean spore load ( $t$ test, $t=1.5$, df $=17, P=$ 0.144 ) nor in the prevalence $(t=0.7$, df $=17, P=$ $0.516)$ between the starved and well-fed populations (Fig. 3). 



FIG. 3. (A) Spore load and (B) prevalence in well-fed and starved Daphnia populations before food reduction treatment (week 21) and 1 and and 6 wk (weeks 22 and 27, respectively) after the food reduction treatment was begun (means $+1 \mathrm{SE}$ ).

$* * P<0.0033$ (levels of significance Bonferroni adjusted for three comparisons); NS, not statistically significant.

For individual animals, the probability of being infected increased with body length at all sampling dates (Fig. 4; change in the log-likelihood if term removed 168.4, df $=1, P<0.003$, odds ratio 1539.7 for week $21 ; 162.1$, df $=1, P<0.003$, odds ratio 256.5 for week 22 and 172.8 , df $=1, P<0.003$, odds ratio 829.9 for week 27; levels of significance Bonferroni adjusted for three tests, $\alpha=0.01 / 3$ ). In weeks 21 and 22 , the food reduction treatment did not influence the probability of infection, only body length did. In week 27 , the effect of food reduction was also highly significant (change in the log-likelihood if term removed 12.6, $\mathrm{df}=1, P$ $<0.003$, odds ratio 0.1 ), indicating that individuals in the starved populations had a lower probability of being infected than those in well-fed populations.

\section{Mortality during food reduction in the population experiment}

The probability of death was significantly higher for the starved than for the well-fed populations in both size classes (Fig. 5, connected with horizontal braces; Kruskal-Wallis $\chi^{2}=19.6$, df $=1, P<0.00125$ for small animals; $\chi^{2}=24.3$, df $=1, P<0.00125$ for large animals; levels of significance Bonferroni adjusted for eight tests, $\alpha=0.01 / 8$ ). In the well-fed populations the probability of death did not differ among size classes (Fig. 5, connected with vertical braces; $\chi^{2}$
$=0.4, \mathrm{df}=1, P=0.545)$, while it did in the starved populations (higher probability of death for the larger hosts; $\chi^{2}=12.0$, df $=1, P<0.00625 ; \alpha=0.05 / 8$ ). As the larger animals carry the highest parasite load, this result is central to our study and can explain the selective removal of parasites under food reduction (compare Fig. 3).

For the small animals, the probability of death did not differ between uninfected and infected animals either in well-fed populations or in starved populations (Fig. 5A, B; $\chi^{2}=0.9$, df $=1, P=0.343$ for well-fed; $\chi^{2}=0.0$, df $=1, P=1.000$ for starved). However, large infected animals in both treatments had a significantly higher probability of death than the uninfected (Fig. 5C, well fed, $\chi^{2}=11.9$, df $=1, P<0.00625$; Fig. 5D, starved, $\chi^{2}=12.9$, df $=1, P<0.00125$ ).

\section{Experiment of time until death under complete starvation}

The mean time to death in complete starvation was $150.1 \pm 58.4 \mathrm{~h}($ mean $\pm \mathrm{SD})$ for the uninfected Daphnia
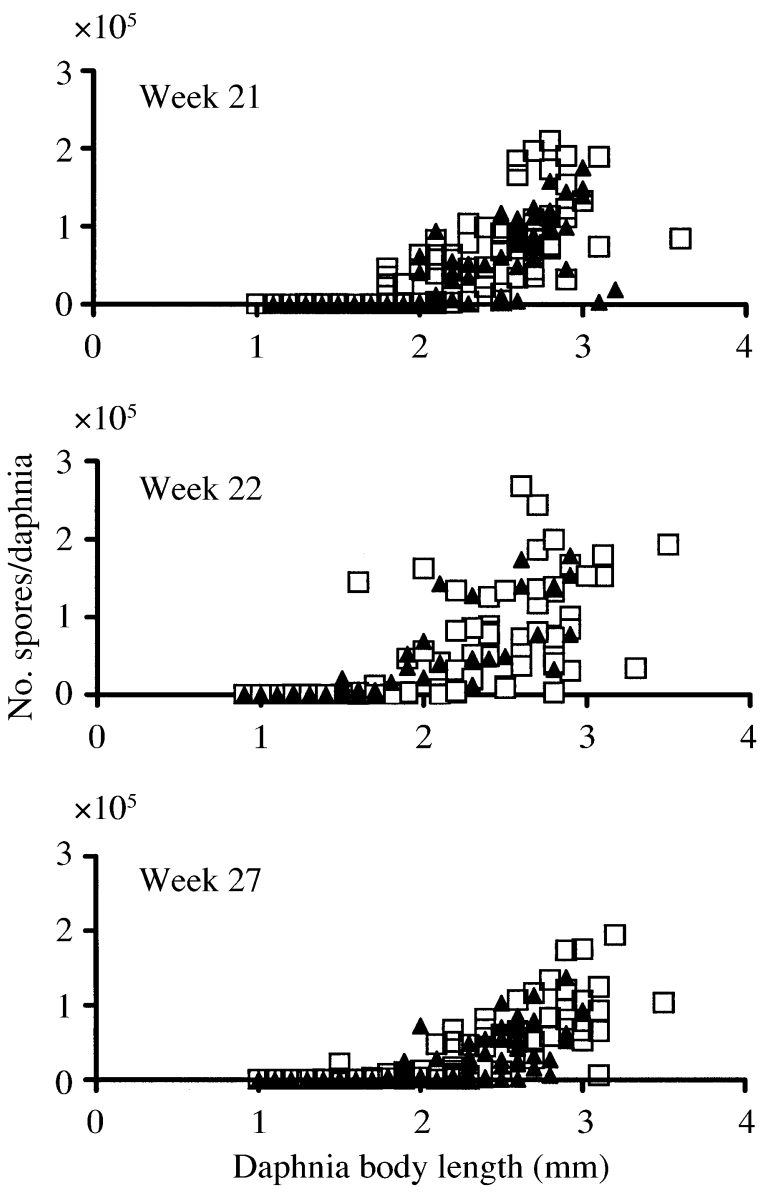

FIG. 4. The relationship between body length and spore load in individual Daphnia in well-fed populations (open squares) and in starved populations (closed triangles) before food reduction treatment (week 21) and 1 and 6 wk after (weeks 22 and 27, respectively) the food reduction treatment was begun. 


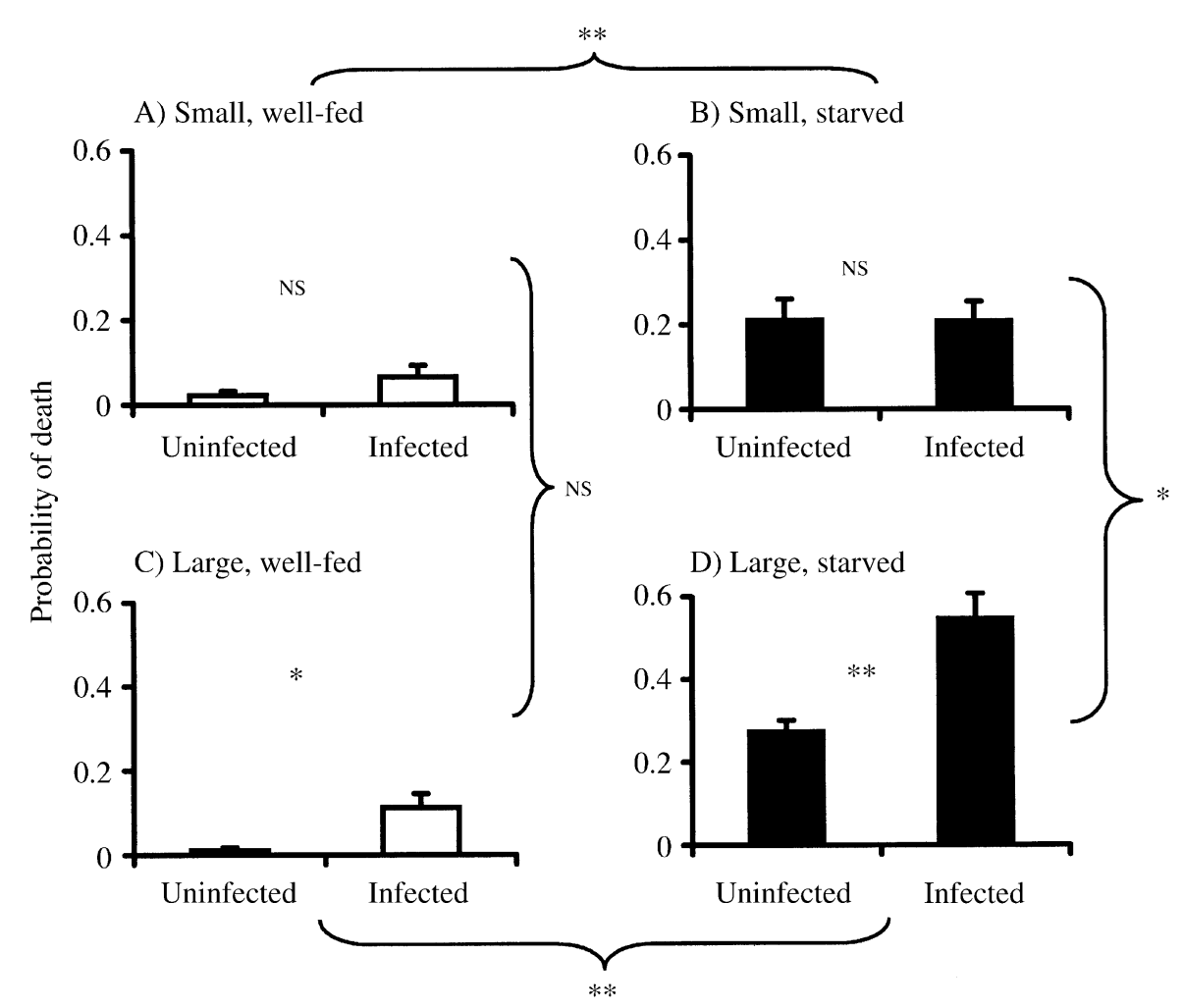

FIG. 5. Probability of death for uninfected and infected Daphnia magna 3-7 d after the beginning of the food reduction treatment in two size classes (small, 0.9-2.0 mm; large, 2.1-3.7 mm) for well-fed and starved populations $(\mathrm{means}+1 \mathrm{SE}$ ).

${ }^{*} P<0.00625 ; * * P<0.00125$ (levels of significance Bonferroni adjusted for eight comparisons); NS, not statistically significant.

and $83.6 \pm 34.3 \mathrm{~h}$ for the infected Daphnia. Small animals survived in complete starvation significantly longer both when uninfected (Fig. 6, Mann-Whitney $U$ test $U=460.5, P<0.005)$ and infected $(U=698.0$, $P<0.005$; levels of significance Bonferroni adjusted for two tests, $\alpha=0.01 / 2$ ). We could not verify the infection status or spore load of the animals in this

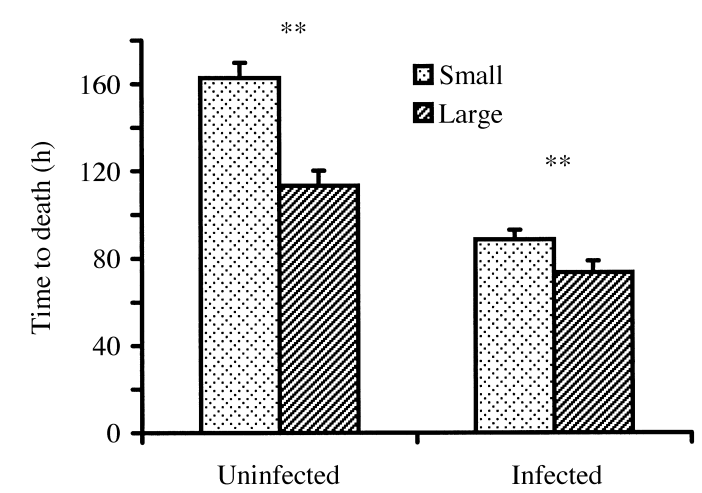

FIG. 6. Starvation times under complete starvation in relation to body length (small, $0.9-2.0 \mathrm{~mm}$; large, $2.1-3.7 \mathrm{~mm}$ ) for Daphnia taken from uninfected and infected populations (means $+1 \mathrm{SE})$.

$* * P<0.005$ (levels of significance Bonferroni adjusted for two comparisons). experiment, but because $G$. intestinalis has high transmission rates, even the very young animals in the infection treatment were probably already infected (Ebert 1995, Ebert and Mangin 1997). The results of this starvation experiment are largely consistent with the results of the death rate analysis (Fig. 5), in that infected hosts tended to die at a higher rate than uninfected hosts and large animals with a higher rate than smaller animals.

\section{Discussion}

Our study shows that food stress in infected Daphnia populations did not facilitate parasite spread. In accordance with our prediction, food shortage caused a disproportionally higher mortality of large animals in the infected populations, which reduced parasite spore load and prevalence (Fig. 3). However, after a few weeks of food reduction, the host population body length distribution regained a shape similar to its pretreatment shape, and parasite spore load increased to approximately the same level as in the well-fed populations. Thus, our results do not support the conventional wisdom that stress leads to an increase in parasitism at the population level.

As mentioned in the introduction, parasite dynamics are influenced by factors that lead to an increase of population size as well as those that lead to a decrease. 
Only their combined effects will reveal the net effect of a treatment. Our results indicate that food stress did not alter the rate at which parasites multiply. If resistance against infection had decreased in individual Daphnia in the populations during food reduction, one would expect spore load to increase for given host size or that the first spores would be detectable in smaller hosts (Fig. 4). We did not find this. On the other hand, if reduced resource level in Daphnia decreased spore production, as has been shown for two other Daphniamicroparasite systems (Ebert et al. 2000b, Bittner et al. 2002), this would result in a decrease in spore loads. We did not find this either. The relationship between body length and spore load in individual Daphnia did not change after the food reduction. In summary, our results suggest that food stress does not alter parasite multiplication rates (within-host growth and transmission), but does lead to a selective removal of strongly infected hosts from the stressed host population. This results in a net (temporary) reduction of parasite population size after the onset of food deprivation.

Lafferty and Holt (2003) make a distinction between effects of stress on the growth rate of the disease $\left(R_{0}\right)$ and impact of disease. They stress that the distinction is important because although $R_{0}$ describes the parasite population growth, it does not represent the impact of the disease on host population growth. The effects of stress on host vital rates may interact with effects of stress on host susceptibility and lead to either a negative, positive, convex, or concave relationship between stress and disease (Lafferty and Holt 2003). In our experiment, the impact of food reduction on the infected host populations was about a $40 \%$ reduction in population size after $1 \mathrm{wk}$ of food deprivation (week 22 ), while in the populations that remained well-fed, there was a $5 \%$ increase in population size. There was a marked decrease in $R_{0}$ for both well-fed and starved populations from week 21 to week 22, but in the starved populations this reduction was clearly larger than in the well-fed populations (Figs. 1A and 3). Thus in our experiment the effects of food stress seem to fall to the left upper corner of Fig. 2 in Lafferty and Holt (2003), i.e., food stress decreased $R_{0}$ and increased the impact of disease on the host population.

Previous theories about the role of stress in infected populations were based on combined results from studies on individual hosts (e.g., higher pathogenicity when hosts are under stress) and observations from disease outbreaks in natural populations (e.g., epidemic outbreaks of parasites may happen in high-density populations [Anderson and May 1981]). Our experiment is the first to test experimentally for stress effects on the population level. Thus, we are able to link our results to the treatments and exclude confounding factors. However, given the nature of these types of epidemiological experiments in which the treatments define the starting conditions, we cannot draw direct conclusions on cause and effect relationships. For example, the reduction in food rations lead to a decrease in host density. Density may have an effect on the parasites (e.g., reducing transmission), perhaps influencing (even counteracting) other effects of starvation. Likewise, starvation may lead to a change in host behavior that could influence host and parasite dynamics. Behavior of Daphnia has been shown to play an important role in transmission dynamics (Ebert et al. 1997, Decaestecker et al. 2002). Thus, while experimental epidemiology can include secondary order effects on the population level that may not be visible at the individual level, it cannot fully elucidate mechanistic cause and effect relationships. The results of our experiment must be seen within the complex interactions of two antagonistic populations as they occur. Only a combination of population- and individual-level experiments could lead to a complete understanding of hostparasite epidemiology.

Conventional wisdom was largely based on the demonstration that malnutrition can reduce immunocompetence, leading to higher parasite multiplication rates (Wakelin 1989, Lloyd 1995). For some invertebrates the opposite has been suggested, based on the phenotypically plastic response of the hosts to stress factors (Reeson et al. 2000). For example, crowding may lead to an increase in disease resistance, at least in some social insects (Traniello et al. 2002) and outbreak pest species (Wilson et al. 2002). Food limitation may also lead to changes in transmission via altered behavior of the hosts (Washburn et al. 1991). A more detailed inspection of single cause and effect relationships may reveal many other specific interactions between hosts and parasites that are modified by starvation or other stress factors. Thus, the distinction between vertebrates and invertebrates made here is clearly an oversimplification, as certainly differences among individual systems may disguise such a classification.

The results of this paper show that host starvation may have a significant effect on the epidemiology of microparasites in zooplankton populations. In our experiment, food deprivation induced a disproportionally high mortality among the most heavily infected individuals in the populations and thus decreased the parasite load in the host populations significantly. Periods of severe starvation are rather common for plankton populations (e.g., the clear water stage observed in many lakes) and may reduce host density by several orders of magnitude (Wetzel 1994). This is likely to slow down the parasites' transmission temporarily. In our experimental populations, the parasite load returned to prestarvation level in a few weeks, by which time the starved populations had reached a new equilibrium at the new carrying capacity. With more severe starvation, this may take much longer. The increased mortality rate due to food shortage may, however, also lead to an increase in transmission rates. G. intestinalis is known to spread from decaying host cadavers (Ebert 1995), and in our experimental containers, the dead 
cadavers were readily reached by Daphnia. Spores released from decaying hosts can survive in the sediments for extended periods, and in the natural populations of shallow ponds and lakes, animals browsing for food particles on the sediment surface may become infected (Ebert et al. 1997). As this browsing behavior is intensified during starvation periods, it may lead to the start of epidemics in Daphnia inhabiting shallow water bodies (Ebert et al. 1997).

$G$. intestinalis is a microsporidian parasite of the Daphnia gut epithelium. The harm it inflicts on its host is rather minimal compared to other Daphnia parasites (Ebert et al. 2000a). Consistent with this, it hardly influenced host density (Fig. 1; see also Ebert et al. $[2000 a]$ ). Its low virulence allows it to be maintained without intervention for long periods in relatively small laboratory cultures. It is very common in natural populations (Larsson et al. 1996, Stirnadel and Ebert 1997, Decaestecker 2002) and often reaches prevalence near $100 \%$ among the adult hosts. Our study shows that the apparent low virulence of this parasite can nevertheless have a profound impact on the size distribution of the host population, which reflects on the patterns of abundance of the parasite itself. There are many more parasites and epibionts of plankton organisms (Green 1974, Threlkeld et al. 1993) that appear harmless but that may show similar effects once properly investigated.

\section{Effect of food stress on survival}

Large infected and uninfected animals died sooner under starvation than small ones both in our population experiments and when individual animals were exposed to complete starvation. This was an unexpected result, as earlier laboratory studies found that small (uninfected) Daphnia survive less under starvation than larger animals (Tessier and Goulden 1982, 1987, Tessier et al. 1983, Tessier and Consolatti 1989, Perrin et al. 1992). This result has been regarded as universally valid among cladocerans and used to predict outcome of competition (Matveev and Gabriel 1994). However, some field studies have recently questioned this contention and indicated adult mortality is a major component in competitive exclusion (Matveev and Gabriel 1994) and midsummer decline in Daphnias (Hülsmann and Weiler 2000). In contrast to our study, individually grown animals in previous laboratory studies were acclimated to high food concentrations prior to starvation. In our experiment, Daphnia were kept in populations around the carrying capacity, i.e., at rather low food levels, before the starvation treatments, so they may have been preadapted to low resource levels. Our results therefore conform to the field data (Matveev and Gabriel 1994, Hülsmann and Weiler 2000), rather than to earlier laboratory studies (Tessier and Goulden 1982).

\section{ACKNOWLEDGMENTS}

We thank Urs Stiefel, Jürgen Hottinger, and the students at the Zoological Institute for help during the experimental work and Kevin Lafferty and an anonymous referee for helpful comments on the manuscript. K. Pulkkinen was supported by the Academy of Finland. The work was supported by the Swiss Nationalfonds.

\section{Literature Cited}

Anderson, R. M. 1978. The regulation of host population growth by parasitic species. Parasitology 76:119-157.

Anderson, R. M. 1979a. The influence of parasitic infection on the dynamics of host population growth. Pages 245281 in R. M. Anderson, B. D. Turner, and L. R. Taylor, editors. Population dynamics. Blackwell Scientific, Oxford, UK

Anderson, R. M. 1979b. Parasite pathogenity and the depression of host population equilibria. Nature 279:150152.

Anderson, R. M., and R. M. May. 1978. Regulation and stability of host-parasite population interactions. I. Regulatory processes. Journal of Animal Ecology 47:219-247.

Anderson, R. M., and R. M. May. 1979. Population biology of infectious diseases. Part I. Nature 280:361-367.

Anderson, R. M., and R. M. May. 1981. The population dynamics of microparasites and their invertebrate hosts. Philosophical Transactions of the Royal Society of London B 291:451-524.

Bittner, K., K.-O. Rothhaupt, and D. Ebert. 2002. Ecological interactions of the microparasite Caullerya mesnili (Chatton 1907) and its host Daphnia galeata from a large lake. Limnology and Oceanography 47:300-305.

Briggs, C. J., R. S. Hails, N. D. Barlow, and H. C. J. Godfray. 1995. The dynamics of insect-pathogen interactions. Pages 295-326 in B. T. Grenfell, editor. Ecology of infectious diseases in natural populations. Cambridge University Press, Cambridge, UK.

Decaestecker, E. 2002. Evolutionary ecology of host-parasite interactions: Daphnia and its parasites as a model. Dissertation. Katholieke Universiteit Leuven, Leuven, Belgium.

Decaestecker, E., L. De Meester, and D. Ebert. 2002. In deep trouble: habitat selection constrained by multiple enemies in zooplankton. Proceedings of the National Academy of Sciences of the United States of America 99:5481-5485.

Ebert, D. 1994. Virulence and local adaptation of a horizontally transmitted parasite. Science 265:1084-1086.

Ebert, D. 1995. The ecological interactions between a microsporidian parasite and its host Daphnia magna. Journal of Animal Ecology 64:361-369.

Ebert, D., C. Haag, M. Kirkpatrick, M. Riek, J. W. Hottinger, and V. I. Pajunen. 2002. A selective advantage to immigrant genes in a Daphnia metapopulation. Science 295: 485-488.

Ebert, D., J. W. Hottinger, and I. Pajunen. 2001. Temporal and spatial dynamics of parasite richness in a Daphnia metapopulation. Ecology 82:3417-3434.

Ebert, D., M. Lipsitch, and K. L. Mangin. 2000a. The effect of parasites on host population density and extinction: experimental epidemiology with Daphnia and six microparasites. American Naturalist 156:459-477.

Ebert, D., and K. L. Mangin. 1997. The influence of host demography on the evolution of virulence of a microsporidian gut parasite. Evolution 51:1828-1837.

Ebert, D., R. J. H. Payne, and W. W. Weisser. 1997. The epidemiology of parasitic disease in Daphnia. Pages 91111 in E. Dettner, editor. Vertical food web interactions. Springer-Verlag, Berlin, Germany.

Ebert, D., C. D. Zchokke-Rohringer, and H. J. Carius. $2000 \mathrm{~b}$ Dose effects and density-dependent regulation of two microparasites of Daphnia magna. Oecologia 122:200-209. 
Ghilarov, A. M. 1985. Dynamics and structure of cladoceran populations under conditions of food limitation. Archiv für Hydrobiologie Ergebnisse der Limnologie 21:323-332.

Green, J. 1974. Parasites and epibionts of Cladocera. Transactions of the Zoological Society of London 32:417-515.

Gulland, F. M. D. 1992. The role of nematode parasites in Soya sheep (Ovis-aries L) mortality during a population crash. Parasitology 105:493-503.

Gulland, F. M. D. 1995. The impact of infectious diseases on wild animal populations-a review. Pages 20-51 in B. T. Grenfell and A. P. Dobson, editors. Ecology of infectious disease in natural populations. Cambridge University Press, Cambridge, UK.

Hülsmann, S., and W. Weiler. 2000. Adult, not juvenile mortality as a major reason for the midsummer decline of a Daphnia population. Journal of Plankton Research 22:151168.

Klüttgen, B., M. Dülmer, M. Engels, and H. T. Ratte. 1994. ADaM, an artificial freshwater for the culture of zooplankton. Water Research 28:743-746.

Lafferty, K. D., and R. D. Holt. 2003. How should environmental stress affect the population dynamics of disease? Ecology Letters 6:654-664.

Lampert, W., and P. Muck. 1985. Multiple aspects of food limitation in zooplankton communities: the Daphnia-Eudiaptomus example. Archiv für Hydrobiologie Ergebnisse der Limnologie 21:311-322.

Larsson, J. I., D. Ebert, J. Vavra, and V. N. Voronin. 1996. Redescription of Pleistophora intestinalis Chatton, 1907, a microsporidian parasite of Daphnia magna and Daphnia pulex, with establishment of the genus Glugoides (Microspora, Glugeidae). European Journal of Protistology 32: 251-261.

Lloyd, S. 1995. Environmental influences on host immunity. Pages 327-361 in B. T. Grenfell and A. P. Dobson, editors. Ecology of infectious diseases in natural populations. Cambridge University Press, Cambridge, UK.

Mangin, K. L., M. Lipsitch, and D. Ebert. 1995. Virulence and transmission modes of two microsporidia in Daphnia magna. Parasitology 111:133-142.

Matveev, V., and W. Gabriel. 1994. Competitive exclusion in Cladocera through elevated mortality of adults. Journal of Plankton Research 16:1083-1094.

May, R. M., and R. M. Anderson. 1979. Population biology of infectious diseases. Part II. Nature 280:455-461.

Perrin, N., D. J. Baird, and P. Calow. 1992. Resource allocation, population dynamics and fitness: some experiments with Daphnia magna Straus. Archiv für Hydrobiologie 123: 431-449.
Potvin, C., and M. J. Lechowicz. 1990. The statistical analysis of ecophysiological response curves obtained from experiments involving repeated measures. Ecology 71:13891400.

Reeson, A. F., K. Wilson, J. S. Cory, P. Hankard, J. M. Weeks, D. Goulson, and R. S. Hails. 2000. Effects of phenotypic plasticity on pathogen transmission in the field in the Lepidoptera-NPV system. Oecologia 124:373-380.

Slobodkin, L. B. 1954. Population dynamics in Daphnia obtusa Kurtz. Ecological Monographs 24:69-88.

SPSS. 1999. SPSS for Windows. Release 10.0.0. SPSS, Chicago, Illinois, USA.

Stirnadel, H. A., and D. Ebert. 1997. Prevalence, host specificity and impact on host fecundity of microparasites and epibionts in three sympatric Daphnia species. Journal of Animal Ecology 66:212-222.

Tessier, A. J., and N. L. Consolatti. 1989. Variation in offspring size in Daphnia and consequences for individual fitness. Oikos 56:269-276.

Tessier, A. J., and C. E. Goulden. 1982. Estimating food limitation in cladoceran populations. Limnology and Oceanography 27:707-717.

Tessier, A. J., and C. E. Goulden. 1987. Cladoceran juvenile growth. Limnology and Oceanography 32:680-686.

Tessier, A. J., L. L. Henry, and C. E. Goulden. 1983. Starvation in Daphnia: energy reserves and reproductive allocation. Limnology and Oceanography 28:667-676.

Threlkeld, S. T., D. A. Chiavelli, and R. L. Willey. 1993. The organization of zooplankton epibiont communities. Trends in Ecology and Evolution 8:317-321.

Traniello, J. F. A., R. B. Rosengraus, and K. Savoie. 2002. The development of immunity in a social insect: evidence for the group facilitation of disease resistance. Proceedings of the National Academy of Sciences of the United States of America 99:6838-6842.

von Ende, C. N. 1993. Repeated-measures analysis: growth and other time-dependent measures. Pages 113-137 in S. M. Scheiner and J. Gurevitch, editors. Design and analysis of ecological experiments. Chapman and Hall, New York, New York, USA.

Wakelin, D. 1989. Nature and nurture: overcoming constraints on immunity. Parasitology 99:S21-S35.

Washburn, J. O., D. R. Mercer, and J. R. Anderson. 1991. Regulatory role of parasites: impact on host population shifts with resource availability. Science 253:185-188.

Wetzel, R. G. 1994. Limnology. Second edition. Saunders College Publishing, Philadelphia, Pennsylvania, USA.

Wilson, K., M. B. Thomas, S. Blanford, M. Doggett, S. J. Simpson, and S. L. Moore. 2002. Coping with crowds: density-dependent disease resistance in desert locusts. Proceedings of the National Academy of Sciences of the United States of America 99:5471-5475. 